\title{
Sentiment Scoring and Performance Metrics Examination of Various Supervised Classifiers
}

\author{
Sherin Mariam John, K. Kartheeban
}

\begin{abstract}
Sentiment Analysis probes public opinion on user generated content on Web like blogs, social media or e-commerce websites. The results of Sentiment Analysis are getting much attention with marketers that they are able to evaluate the success of an advertising campaign or the attitude of people on a new product launch. Business owners and advertising companies are using Sentiment Analysis to start new business strategies and to identify opportunities for new product development. In this paper, with $R$ programming, the tweets from Twitter about Samsung Galaxy mobile phone and Apple Iphone were retrieved from three countries namely USA, UK and India for creating the dataset. The collected tweets were classified into positive, negative and neutral sentiments. The machine learning classifier algorithms like Nä̈ve Bayes, Support Vector Machine, Random Forest, Decision Tree, Artificial Neural Network, XGBoost with $K$ Fold cross validation were applied on the dataset and the results were tabulated for comparing and estimating which classifier algorithm yields the best accuracy. Other performance metric values like F Score, Precision, Recall were also calculated for comparison of various classifier performances on Sentiment Analysis. It was found that XGBoost method combined with $K$ Fold cross validation has produced the best accuracy in prediction. We have also applied SentiStrength algorithm to find out the intensity or the strength of positive and negative comments from each sentence. With the help of the results in hand, we were able to predict the brand of mobile phone that was preferred in each country.
\end{abstract}

Keywords: Sentiment Analysis, Machine learning, Text Mining and Analytics, Web Data Mining, Predictive Analytics

\section{INTRODUCTION}

Sentiment Analysis helps us to know about the public opinion of a product. For instance, prior to purchase of a product most people are likely to search through the reviews about that product which will help them in making a choice. E-Commerce is growing rapidly [14] and the $\mathrm{Z}$ generation is more interested in buying online than going to a physical store for purchase. The online reviews are treated with much importance before purchase. Our competition is with the global market and a single global rating could change the perspective regarding a product.

Sentiment analysis can be done at different levels of granularity - word/word-phrase/aspect level, sentence level and document level. The strength of adjectives and adverbs in the reviews are taken for estimating the sentiment strength score [20].

Revised Manuscript Received on December 15, 2019

* Correspondence Author

Sherin Mariam John*, Computer Science Engineering Department

Kalasalingam Academy of Research and Education Krishnankoil, India

sherinmjohn@gmail.com

K. Kartheeban, Computer Science Engineering Department,

Kalasalingam Academy of Research and Education Krishnankoil, India k.kartheeban@klu.ac.in
The words like "super", "excellent" have more strength than "good", "nice". The sentiment value also changes according to the context in which it is expressed. For example the sentence "How I wish the battery could last longer" is actually a negative comment on the aspect of battery charge. The understanding of the context in which the comment is written is a research challenge.

Sentiment Analysis is one of the applications of Text Mining and Analytics coming under Natural Language Processing. The analysis of text data is more complex and tedious than digital data analysis. Text mining is different from data mining in that the data source for text mining is unstructured, whereas the data source for data mining is structured. Text Mining techniques can be classified into three major categories namely Descriptive, Predictive and Prescriptive. The descriptive text mining processes raw data and convert it to meaningful information. It assists users in finding the optimal solution to a problem. $80 \%$ of text mining applications are descriptive.

Predictive analytics apply a variety of statistical, modeling, and machine learning techniques to study recent and historical data. It predicts the probability of occurrence of a future event and helps organizations to plan their future course of action. This kind of analysis is the most frequently used across several industries. Prescriptive data analytics recommends one or two course of action for decision making.

The concept of Machine Learning is similar to the way human beings learn from their past experiences and use it to solve present day problems. There are two types of learning's in Machine learning namely supervised learning and unsupervised learning. Classification algorithms come under supervised learning and our research is focused on implementing various classification methods for classifying tweets regarding an entity and identifies the public opinion about that entity.

Given an opinion document $\mathrm{d}$, tweets retrieved from Twitter, classify $\mathrm{d}$ into positive, negative or neutral sentiments. Compare and determine the best classifier for prediction based on accuracy and performance metrics values.

Evaluating a classifier for its accuracy is very important because without knowing the efficiency of a classifier in prediction, it cannot be used in real world tasks. Precision and Recall are the best suitable evaluation methods for text applications. They measure how precise and complete the classification has been done. 
A confusion matrix contains information about actual and predicted results given by a classifier.

\section{RELATED WORK}

The three approaches for performing sentiment extraction are (i) machine learning approach (ii) lexicon-based approach and (iii) hybrid approach by combining machine learning and lexicon-based [23].

Machine learning approach to sentiment analysis can be categorized into two ways: Supervised learning and unsupervised learning approach [11].

Supervised learning involves classifier and training set of data whereas the Unsupervised learning method involves a classifier which works on the association principle. The classifier compares the characteristics of a given text against the sentiment lexicons whose sentiment value is already known. After comparison the given text is classified to the category of the lexicon with which it matches and respective sentiment value is assigned to it.

Data collected from Twitter website is subjected to Sentiment Analysis using Naïve Bayes Classifier and predicted user's age and gender. Analyzed the data based on various consumer parameters such as location, gender and age group [18]. Lexicon based methods were designed to overcome the difficulty in training and labeling the data in Supervised learning algorithms. These methods use a dictionary of sentiment words called lexicons to match the words in the tweet and calculate the sentiment orientation [12].

Micro blogging feature of Twitter website allows users to text messages, send images, audio or URL. Expressing emotions using Emoticons is a common practice in Twitter but difficult to analyze [13]. The research on Sentiment analysis concentrates on the topic of discussion and polarity detection. The modern consumers will go through the customer's review on products they wish to buy prior to purchasing that product. Many websites are available which discusses about the good and bad features of the product that comes to market. Therefore, it is very much needed for the marketing people to understand the sentiments of the public so that they can go for branding, market segmenting and product penetration [16].

To detect the strength of sentiments from text written in English, we can use the algorithm SentiStrength which uses linguistic information. It is a classifier based on lexicon $[19,16]$. The output from this classifier algorithm can be measured on a scale of -5 to +5 . The negative sentiments are given a score which is below zero and positive sentiments are given a score of above zero [19].

Ensemble method decomposes a data set into many overlapping data samples and consolidates the results got from various base classifiers. The application of correct weightage to the different words in a sentence is very important feature in sentiment analysis and this was achieved in neural models [1].

\section{CLASSIFICATION METHODS - A} THEORETICAL OUTLINE
The process of classifying tweets into positive and negative sentiments can be treated as a classification problem. Hence we can employ different classifiers for the task and verify the performance of these classifiers by calculating Accuracy of prediction. Other performance metrics like Precision, Recall, F Score are also determined. These parameters are calculated from Confusion Matrix.

Although many classification models or otherwise we can call as classifiers are available, we have chosen the classifiers suitable for text analysis. An important measure of evaluating the classifier performance is through accuracy detection. The accuracy of a classifier can be determined as follows:

$$
\text { Accuracy }=\frac{\text { Number of records classified correctly }}{\text { count of entire data records }}
$$

In addition to accuracy, we need other performance metrics like Precision, Recall and F measure for evaluating classifier performance, since in some cases accuracy alone will not yield the correct result that we opt for. The actual and predicted results given by a classifier can be represented in a confusion matrix as shown in Table 1 and the values can be used for calculations as per formulas given below.

Accuracy $=(\mathrm{TP}+\mathrm{TN}) /(\mathrm{TP}+\mathrm{TN}+\mathrm{FP}+\mathrm{FN})$

Precision (measuring exactness $)=\mathrm{TP} /(\mathrm{TP}+\mathrm{FP})$

Recall (measuring completeness $)=\mathrm{TP} /(\mathrm{TP}+\mathrm{FN})$

$\mathrm{F} 1=(2 *$ Precision $*$ Recall $) /($ Precision + Recall $)$

Table I. The format of a confusion matrix.

\begin{tabular}{|c|l|l|}
\hline \multirow{2}{*}{ Actuals } & Predicted class \\
\cline { 2 - 3 } & Positive Forecasts & $\begin{array}{l}\text { Negative } \\
\text { Forecasts }\end{array}$ \\
\hline Actual Positive & TP(Real Positives) & FN (Wrong \\
& & Negatives) \\
\hline Actual Negative & FP(Wrong & TN (Real \\
& Positives) & Negatives) \\
\hline
\end{tabular}

The F measure is a harmonic mean of Precision and Recall. Sometimes it becomes difficult to take a decision based on Precision and Recall only when one of their values goes very high or very low. Then we can decide upon the validity of the result by considering the $\mathrm{F}$ measure.

\section{A. Nä̈ve Bayesian Text Classifier}

The bag of words model is applied in Naïve Bayesian classification. Naïve Bayes is a probability function which identifies the possible classes to which a document belongs and it is based on Bayes theorem. The Bayes theorem is based on

probability theory and is formulated as:

$\operatorname{Prb}(\mathrm{A} / \mathrm{B})=(\operatorname{Prb}(\mathrm{B} / \mathrm{A}) * \operatorname{Prb}(\mathrm{A})) / \operatorname{Prb}(\mathrm{B})$

Here $A$ can be any event based on entity $B$. All the probabilities needed for the classification are calculated by scanning the data records only once. The same probability set or a revised version can be used for the classification model when new dataset is tested. The model is thus easily updated. 
Let $\mathrm{v}=\left\{\mathrm{w}_{1}, \mathrm{w}_{2}, \ldots \ldots, \mathrm{w}_{\mathrm{n}}\right\}$ be the vector containing all the words

in the document and $\mathrm{C}=\left\{\mathrm{c}_{1}, \mathrm{c}_{2}, \ldots . . \mathrm{c}_{\mathrm{m}}\right\}$ be the probability

classes. The formula for classification is as follows:

$$
\operatorname{Pr}\left(\mathrm{c}_{\mathrm{i}} / \mathrm{w}_{\mathrm{j}}\right)=\operatorname{Pr}\left(\mathrm{w}_{\mathrm{j}} / \mathrm{c}_{\mathrm{i}}\right) * \operatorname{Pr}\left(\mathrm{c}_{\mathrm{i}}\right) / \operatorname{Pr}\left(\mathrm{w}_{\mathrm{j}}\right)
$$

\section{B. Support Vector Machine (SVM)}

SVM is a very popular classifier among researchers since it performs classification more accurately than other algorithms. It has a strong theoretical foundation and is widely used for text classification. SVM is a linear learning system. The classifier is modeled based on the equation

$$
\mathrm{fn}(\mathrm{x})=\left(\mathrm{w}^{*} \mathrm{x}\right)+\mathrm{b}
$$

An input vector $x$ is assigned to a positive class if $f n(x)>=0$ and to negative class if $f(x)<0$.

\section{Artificial Neural Networks}

Deep Learning is artificial neural networking with many hidden layers. Deep Learning model is nothing but a classification model. Artificial Neural Networks (ANN) improves accuracy prediction through hidden layers. The hidden layers give power and increases accuracy. The input weights in the hidden layers are adjusted to produce maximum output.

\section{D. $\quad$ K Fold Cross Validation}

In $\mathrm{K}$ fold cross validation method, all records in the dataset are taken for both training and testing and each observation is taken for validation or testing exactly once. The whole dataset is divided into $\mathrm{K}$ subsamples. The value of $\mathrm{K}$ can be determined by us and it was found that 10 is the ideal number. From the $\mathrm{K}$ subsamples, 1 sample is used for validation and the remaining K-1 samples are used for training data. This process is repeated $\mathrm{K}$ times by changing the subsample each time for testing. The $\mathrm{K}$ results that obtained can be averaged and made into a single estimated value.

\section{E. Ensemble of Classifiers}

In Ensemble method, many classifiers are built and combined to increase the accuracy of prediction. Bagging and Boosting are two well-known ensemble techniques. Many

algorithms have been developed under these techniques and we have used XGBoost algorithm for our work.

\section{EXPERIMENTAL SETUP}

The first task in hand was to generate the dataset for classifiers. The tweets were collected programmatically by giving Samsung Galaxy and Apple Iphone as search keywords. The collected tweets need to be cleaned because of the casual way of writing tweets. The redundant data also need to be eliminated. This is called as pre-processing stage. Preprocessing refines miscellaneous text into analyzable units of text. The main procedures involved in pre-processing are normalization, tokenization, lemmatization, stemming and stop word removal. Normalization helps improve the quality of the text and produce reliable output. Tokenization is a pre- processing step where the input text is automatically divided into units called tokens. The goal of lemmatization is to conduct proper reduction of words to dictionary word form. Stemming is a process of transforming a word into its normalized form. The general application of stop word removal method is to remove the tokenized word if it is matched with one of the stop words in the stop word list. After the preprocessing is over, the next stage is application of classifier algorithms to find facts and events of interest to users.

\section{A. Dataset Generation and Sentiment Strength calculation}

The algorithm for generating the dataset file with .csv extension, classifying tweets into positive, negative or neutral and calculating the sentiment strength of each tweet is given below.

Step 1: Establish a connection with Twitter dataset.

Step 2: Harvest tweets by giving location of the city and product name.

Step 3: Clean the harvested Tweets by removing symbols, hash tags, URL etc.

Step 4: Extract lexicons.

Step 5: Compare each word from tweet with a collection of positive and negative words

Step 6: Count the hits of positive words and negative words. Step 7: Find a score which predicts the strength of positive or negative sentiment.

Step 8: Sentiment Strength score is got by summing up the positive and negative score.

Step 9: Create a data frame in excel with .csv as extension.

The dataset generation steps were repeated separately for each country and mobile phones.

The workflow is depicted in Figure 1. The dataset generated is used for analysing the different classifier algorithms and for variance analysis in SPSS tool and also for calculating the sentiment strength of the positive and negative tweets.

\section{RESULTS AND DISCUSSION}

Our first objective was to find out the popularity of the brands Apple Iphone and Samsung Galaxy mobile phones across India, UK and USA. It was found that the Samsung Galaxy mobile phone is more popular in India and Apple Iphone is more talked about positively in UK. We have done analysis in SPSS to support the conclusion. Table 3 indicates the mean difference scores of positive, neutral and negative categories across the three countries. In the case of Samsung Galaxy, the scores of positive reviews, neutral reviews, and negative reviews significantly differ across the countries in $95 \%$ confidence level. But in case of Apple Iphone, the brand scores except for negative reviews do not differ significantly across countries. In the context of sentiment analysis, this will add value to the brand managers to measure the performance of their brand equity in international standards. Moreover, this will allow marketers to know the brand sentiments across different countries.

The results are graphically visualized using Tableau Visualization tool. 
Polarity is calculated as Positive for sentiment score greater than zero, negative for sentiment score less than zero and Neutral for Sentiment Score equal to zero. Table 2 shows the polarity calculation got from Tableau Visualization tool. It is graphically represented in Figure 2.

\section{A. Comparative Study on Text Classification Methods}

The tweets are cleaned for machine learning models. A bag of word model was created from the pre-processed tweets. The machine learning algorithms are applied to this bag of words. The training set data and test data were divided in the ratio $75: 25$.

SVM has yielded a good accuracy over other methods like Naïve Bayesian, Decision Tree, Random Forest and Artificial Neural Network method. But when we combined Boosting algorithm with 10 fold cross validation, the result was high accuracy with better performance.

We were able to boost the value of accuracy by combining Boosting method and 10 fold cross validation. The training set was divided into $\mathrm{k}=10$ folds. Each fold(iteration) is divided into training set and test set in the ratio 80:20. For each iteration, the accuracy is calculated. The average of 10 accuracies was taken (96.75) and it was the highest accuracy we got when compared to all other classifiers.

The benefits of 10 fold validation and Boosting were improved speed of execution, suitable for large dataset and improved accuracy. Table 4 shows the accuracies compiled for the various classifiers. Table 5 illustrates the values of Precision, Recall and F measure. The high values of Precision and Recall shows that the performances of all the classifiers except Naive Bayesian are very good.

Figure 3 shows the graphical representation of various classifier accuracies and it can be seen that the XGBoost algorithm combined with $\mathrm{K}$ Fold iterations yielded the highest accuracy. Figure 4 shows a high value of Precision and Recall for different classifiers showing a high performance for all classifiers except Naïve Bayes.

\section{CONCLUSION AND FUTURE WORK}

One of the goals of this research was to find out the popularity of the smart phone brands in various geographical regions which helps managers to position their product in the market. The dataset for the research was retrieved online from Twitter specifying the geographic location of cities in a country; time period and the keywords used for search were Samsung Galaxy and Apple Iphone. The dataset was used for discovering about the more discussed brand positively in each country. The dataset was also used for study of various classifier performances. Of all the classifiers we found that XGBoost combined with $\mathrm{K}=10$ fold validation yielded the best accuracy in prediction.

The SentiStrength algorithm was also implemented to find the strength of positive comment and the negative comment from each tweet. Further research should focus on challenges like Sarcasm in tweets, Short forms used while writing and taking care of spelling mistakes and spams in tweets.

Table II. Depicting Classifier Accuracy for Different Classifiers

\begin{tabular}{|c|c|}
\hline Classifiers & Accuracy(\%) \\
\hline $\begin{array}{c}\text { XGBoost algorithm combined with } \\
10 \text { fold cross validation }\end{array}$ & $\mathbf{9 6 . 7 5}$ \\
\hline Support Vector Machine & 95.75 \\
\hline Decision Tree & 93.00 \\
\hline Random Forest & 93.25 \\
\hline Artificial Neural Nework & 93.25 \\
\hline Naïve Bayesian Text Classifier & 40.50 \\
\hline
\end{tabular}

Table III. The study of performance metrics with various classifiers

\begin{tabular}{|c|r|r|r|}
\hline Classifiers & Precision & Recall & F Score \\
\hline SVM & 0.94 & 0.98 & 0.96 \\
\hline Decision Tree & 0.90 & 0.99 & 0.94 \\
\hline Random Forest & 0.91 & 0.98 & 0.94 \\
\hline $\begin{array}{c}\text { Artificial Neural } \\
\text { Nework }\end{array}$ & 0.95 & 0.94 & 0.94 \\
\hline Naive Bayes & 0.10 & 0.12 & 0.21 \\
\hline
\end{tabular}

Table IV. The polarity of samsung mobile phone and iphone across countries india, uk and usa.

\begin{tabular}{|c|l|l|l|l|l|l|}
\hline \multirow{2}{*}{} & \multicolumn{2}{|c|}{ Country / Product } & \multicolumn{2}{c|}{ UK } & \multicolumn{2}{c|}{ US } \\
\cline { 2 - 7 } Polarity & \multicolumn{2}{|c|}{ India } & Samsung & Iphone & Samsung & \multicolumn{2}{c|}{ Iphone } & Samsung \\
\cline { 2 - 7 } & Iphone & $2.13 \%$ & $10.08 \%$ & $5.65 \%$ & $10.33 \%$ & $5.53 \%$ \\
\hline Negative & $10.50 \%$ & $28.13 \%$ & $40.28 \%$ & $65.30 \%$ & $62.55 \%$ & $62.87 \%$ \\
\hline Neutral & $61.88 \%$ & $69.75 \%$ & $49.65 \%$ & $29.05 \%$ & $27.13 \%$ & $31.60 \%$ \\
\hline
\end{tabular}


International Journal of Innovative Technology and Exploring Engineering (IJITEE) ISSN: 2278-3075, Volume-9 Issue-2S2, December 2019

Table V. Results of variance analysis c

\begin{tabular}{|c|c|c|c|c|c|c|c|}
\hline \multicolumn{8}{|l|}{ Multiple Comparisons } \\
\hline & & & Games-Howell & & & & \\
\hline \multirow[t]{2}{*}{ Dependent Variable } & \multirow[t]{2}{*}{ (I) Country } & \multirow[t]{2}{*}{ (J) Country } & \multirow{2}{*}{ Mean Difference (I-J) } & \multirow[t]{2}{*}{ Std. Error } & \multirow[t]{2}{*}{ Sig. } & \multicolumn{2}{|c|}{ 95\% Confidence Interval } \\
\hline & & & & & & Lower Bound & Upper Bounc \\
\hline \multirow{6}{*}{ Samsung Positive } & \multirow{2}{*}{ India } & UK & $-62.500^{*}$ & 13.452 & .035 & -117.32 & -7.68 \\
\hline & & US & -3.500 & 28.213 & .992 & -98.30 & 91.30 \\
\hline & \multirow{2}{*}{ UK } & India & $62.500^{*}$ & 13.452 & .035 & 7.68 & 117.32 \\
\hline & & US & 59.000 & 24.942 & .187 & -44.45 & 162.45 \\
\hline & \multirow{2}{*}{ US } & India & 3.500 & 28.213 & .992 & -91.30 & 98.30 \\
\hline & & UK & -59.000 & 24.942 & .187 & -162.45 & 44.45 \\
\hline \multirow{6}{*}{ Samsung Neutral } & \multirow{2}{*}{ India } & UK & $-160.000 *$ & 8.822 & .000 & \begin{tabular}{|l|}
-194.83 \\
\end{tabular} & -125.17 \\
\hline & & US & -24.250 & 24.752 & .627 & -114.51 & 66.01 \\
\hline & \multirow{2}{*}{ UK } & India & $160.000^{*}$ & 8.822 & .000 & 125.17 & 194.83 \\
\hline & & US & $135.750^{*}$ & 23.280 & .020 & 39.30 & 232.20 \\
\hline & \multirow{2}{*}{ US } & India & 24.250 & 24.752 & .627 & -66.01 & 114.51 \\
\hline & & UK & $-135.750^{*}$ & 23.280 & .020 & -232.20 & -39.30 \\
\hline \multirow{6}{*}{ Samsung Negative } & \multirow{2}{*}{ India } & $\mathrm{UK}$ & $-26.750^{*}$ & 3.326 & .004 & -39.25 & -14.25 \\
\hline & & US & .750 & 6.707 & .993 & -21.77 & 23.27 \\
\hline & \multirow{2}{*}{ UK } & India & $26.750^{*}$ & 3.326 & .004 & 14.25 & 39.25 \\
\hline & & US & $27.500^{*}$ & 5.993 & .035 & 3.32 & 51.68 \\
\hline & \multirow{2}{*}{ US } & India & -.750 & 6.707 & .993 & -23.27 & 21.77 \\
\hline & & UK & $-27.500^{*}$ & 5.993 & .035 & -51.68 & -3.32 \\
\hline \multirow{6}{*}{ Iphone Positive } & \multirow{2}{*}{ India } & UK & 12.750 & 7.261 & .271 & \begin{tabular}{|l|}
-10.46 \\
\end{tabular} & 35.96 \\
\hline & & US & 95.000 & 50.297 & .283 & -111.34 & 301.34 \\
\hline & \multirow{2}{*}{ UK } & India & -12.750 & 7.261 & .271 & \begin{tabular}{|l|}
-35.96 \\
\end{tabular} & 10.46 \\
\hline & & US & 82.250 & 50.101 & .356 & -125.33 & 289.83 \\
\hline & \multirow{2}{*}{ US } & India & -95.000 & 50.297 & .283 & -301.34 & 111.34 \\
\hline & & UK & -82.250 & 50.101 & .356 & -289.83 & 125.33 \\
\hline \multirow{6}{*}{ Iphone Neutral } & India & UK & $247.500 *$ & 26.273 & .005 & 139.13 & 355.87 \\
\hline & ninta & US & 139.750 & 113.928 & .511 & -306.55 & 586.05 \\
\hline & UK & India & $-247.500^{*}$ & 26.273 & .005 & \begin{tabular}{|l|}
-355.87 \\
\end{tabular} & -139.13 \\
\hline & C & US & -107.750 & 110.920 & .640 & -570.91 & 355.41 \\
\hline & US & India & -139.750 & 113.928 & .511 & -586.05 & 306.55 \\
\hline & & UK & 107.750 & 110.920 & .640 & -355.41 & 570.91 \\
\hline & India & UK & $-27.750^{*}$ & 6.530 & .030 & \begin{tabular}{|l|}
-51.44 \\
\end{tabular} & -4.06 \\
\hline & Nest & US & 1.250 & 10.535 & .992 & -32.11 & 34.61 \\
\hline Iphone Negative & UK & India & $27.750^{*}$ & 6.530 & .030 & 4.06 & 51.44 \\
\hline & & US & 29.000 & 8.898 & .079 & -5.24 & 63.24 \\
\hline & US & India & -1.250 & 10.535 & .992 & -34.61 & 32.11 \\
\hline & & UK & -29.000 & 8.898 & .079 & -63.24 & 5.24 \\
\hline
\end{tabular}

$*$ The mean difference is significant at the 0.05 level.

Sentiment Scoring and Performance Metrics Evaluation of Various Supervised Classifiers

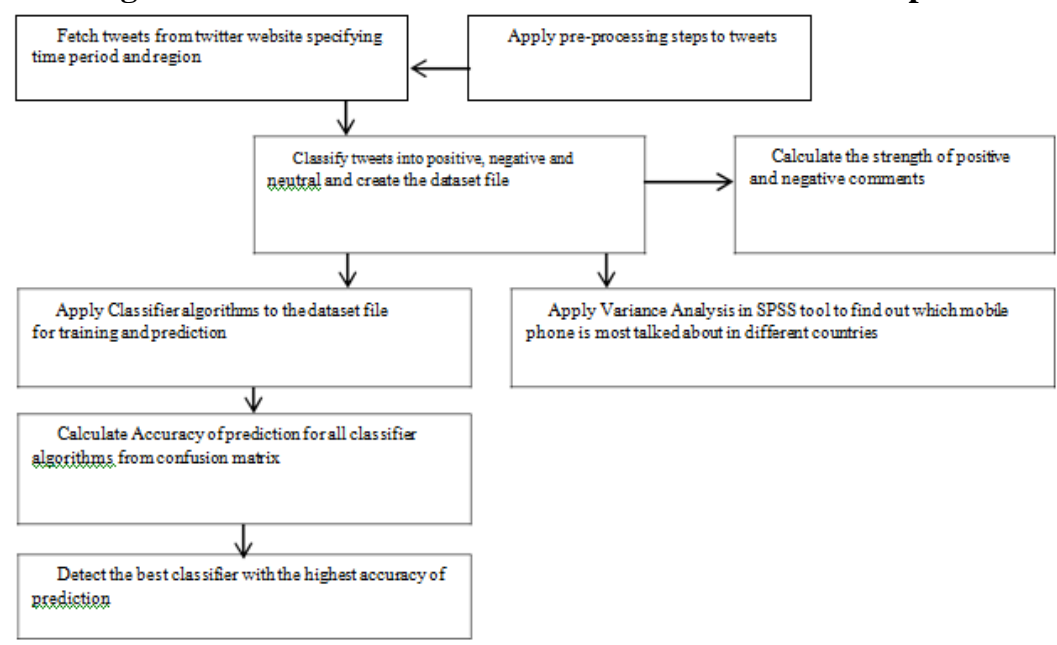


Sentiment Scoring and Performance Metrics Examination of Various Supervised Classifiers

\section{Sentiment Polarity Visualization}

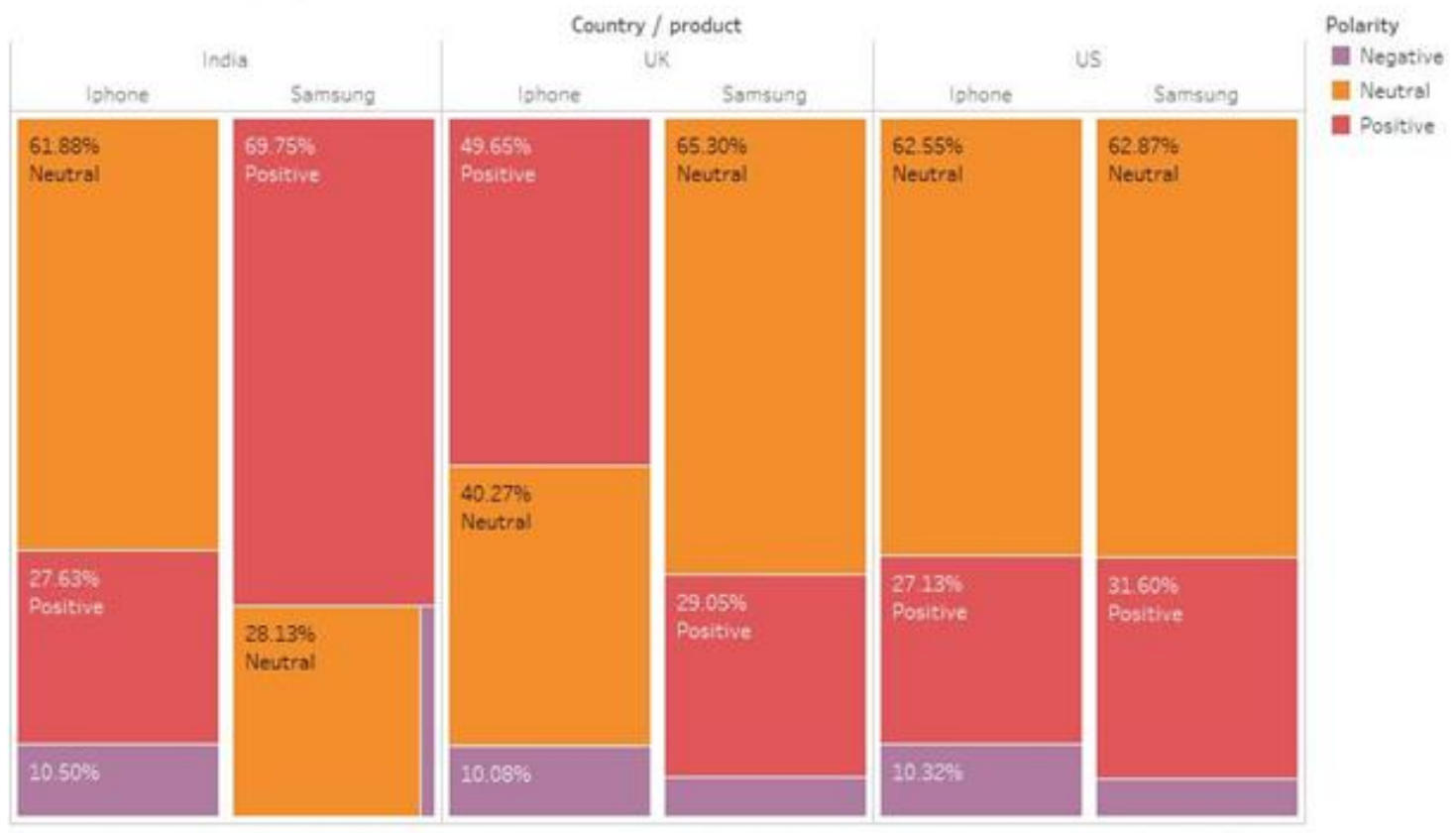

$\%$ of Total Number of Records and Polarity broken down by Country and product. Color shows detalis about Polarity. Size shows \% of Total Number of Records. The marks are labeled by \% of Total Number of Records and Polarity. The data is filtered on duplicate, which keeps Null., Faise and True

Fig. 2 shows the Sentiment Polarity Visualization in Tableau.

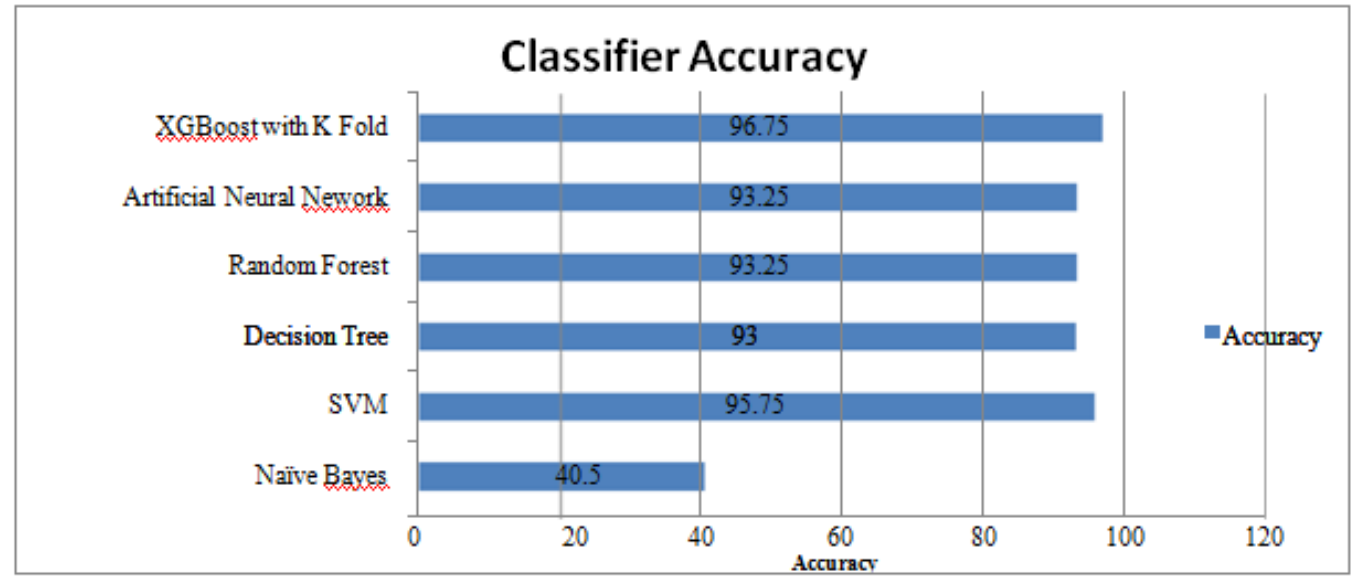

Fig. 3. Chart for comparing different classifier accuracies

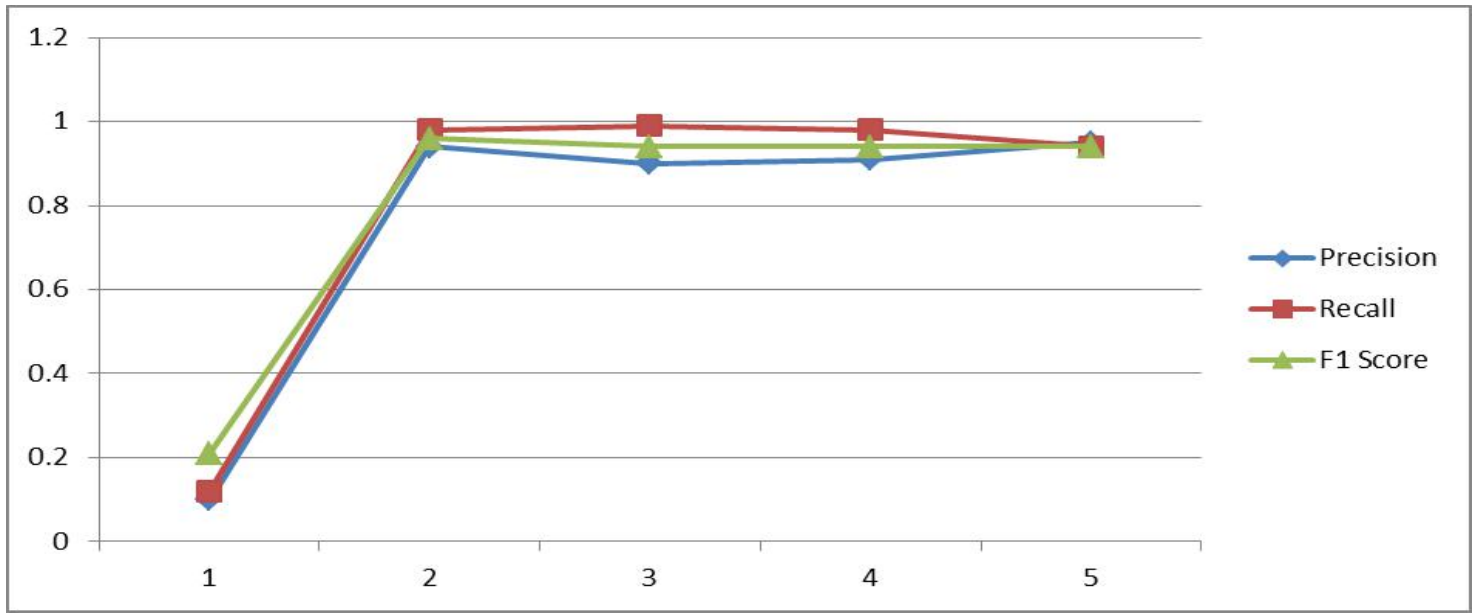

Fig. 4. Chart showing the relationship between Precision and Recall and F1 Score 


\section{REFERENCES}

[1] Zhe Zhao Tao Liu, Guiding the Training of Distributed Text Representation with Supervised Weighting Scheme for Sentiment Analysis,Data Science and Engineering, Volume 2, Issue 2, pp 178186, 2017

[2] Feng, S., Song, K., Wang, D, A word-emoticon mutual reinforcement ranking model for building sentiment lexicon from massive collection of microblogs, World Wide Web, Volume 18, Issue 4, pp 949-967, 2015.

[3] Braja Gopal Patra, A Multilevel Approach to Sentiment Analysis of Figurative Language in Twitter, Springer International Publishing AG, LNCS, Volume 9624, pp. 281-291, 2018.

[4] Ashish Kumar Rathore, Santanu Das, Vigneswara Ilavarasan, Socia Media Data Inputs in Product Design: Case of a Smartphone, Global Journal of Flexible Systems Management, Volume 19, Issue 3, pp 255-272, 2018

[5] Mondher Bouazizi, Tomoaki Ohtsuki, A Pattern-Based Approach for Multi-Class Sentiment Analysis in Twitter, IEEE Access, Volume 5,pp 20617 - 20639, 2017

[6] Kuo, YH., Fu, MH., Tsai, WH., Integrated microblog sentiment analysis from users' social interaction patterns and textual opinions, Applied Intelligence, Springer, Volume 44, Issue 2, pp 399-413, 2016

[7] Liu, H., Cocea, M. \& Ding, W., Multi-task learning for intelligent data processing in granular computing context, Granular Computing, Springer, Volume 3, Issue 3, pp 257-273, 2018.

[8] Lin Yue ,Weitong Chen, A survey of sentiment analysis in social media, Knowledge and Information Systems, Springer, pp 1-47, 2018

[9] Mike Thelwall, The Heart and Soul of the Web? Sentiment Strength

Detection in the Social Web with SentiStrength, Cyberemotions, Springer, pp 119-134, 2017

[10] Andrius Mudinas, Dell Zhang, Mark Levene, Combining Lexicon and Learning based Approaches for Concept-Level Sentiment Analysis, ACM Digital Library,2012

[11] Pranali Borele, Dilipkumar A. Borikar, An Approach to Sentiment Analysis using Artificial Neural Network with Comparative Analysis of Different Techniques, IOSR Journal of Computer Engineering, Volume 18, Issue 2, pp 64-69, 2016

[12] Hassan Saif, Yulan He, Contextual Semantics for Sentiment Analysis of Twitter, Information Processing and Management, Volume 52, Issue 1, pp 5-19, 2016

[13] G. Vaitheeswaran, L. Arockiam, A Novel Lexicon Based Approach to Enhance the Accuracy of Sentiment Analysis on Big Data, International Journal of Emerging Research in Management \&Technology, Volume 5, Issue 1, 2016

[14] A. Nirmala, C. Christy, M.A. Maria Parimala, An Enhanced Sentence Level Sentiment Classification Opinion Mining System With Pos Tagging, International Journal of Emerging Technology in Computer Science \& Electronics, Vol 19, Issue 2, 2016

[15] N.Srinivasa Gupta, B. Valarmathi, Opinion Mining Using An Intuitive Scoring Approach, International Journal of Pharmacy \& Technology, Vol. 8, Issue 4, pp. 21527-21546, 2016

[16] R.Nithyaa,, Dr.D.Maheswari, A Contrast Between Systematic and Automated Sentiment Analysis, I.J. Education and Management Engineering,Vol 2, pp. 20-29, 2015

[17] Fang and Zhan, Sentiment Analysis Using Product Review Data, Journal of Big Data, Vol 2, Issue 5, 2015

[18] A Kowcika, Aditi Gupta, Sentiment Analysis for Social Media, International Journal of Advanced Research in Computer Science and Software Engineering, Vol. 3, Issue 7, 2013

[19] Thelwall, M., Buckley, K., Paltoglou, G., Cai, D., \& Kappas, A., Sentiment strength detection in short informal text, Journal of the American Society for Information Science and Technology, 61(12), pp. 2544-2558, 2010

[20] Lu Y., Kong X., Quan X., Liu W., Xu Y. Exploring the Sentiment Strength of User Reviews, Web-Age Information Management, Vol 6184, pp 471-482, 2010

[21] Bing Liu, Sentiment Analysis and Opinion Mining, Morgan \& Claypool Publishers, May 2012

[22] Bing Liu, Web Data Mining, Springer Publications, 2008

[23] Muhammad Zubair Asghar, T- SAF: Twitter sentiment analysis framework using a hybrid classification scheme, WILEY, 2017

[24] Jaspreet Singh, Optimization of sentiment analysis using machine learning classifiers, Human Centric Computing and Information Sciences, Springer, 2017

[25] Lulu Wang., Weighted Ensemble Classification of Multi-label Data Streams, Springer,2017
[26] Wu He, Zuopeng Zhang \& Vasudeva Akula, Comparing consumerproduced product reviews across multiple websites with sentiment classification, Journal of Organizational Computing and Electronic Commerce, 2018

[27] Cuiqing Jiang, Capturing helpful reviews from social media for product quality improvement: a multi-class classification approach, International Journal of Production Research, 2017

\section{AUTHORS PROFILE}

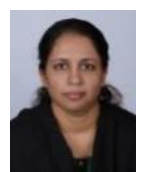

Sherin Mariam John is currently engaged as a $\mathrm{PhD}$ scholar in the Department of Computer Science and Engineering, Kalasalingam Academy of Research and Education, Krishnankoil, Tamilnadu, India. She has completed her masters in Computer Science Engineering from Anna University, Chennai, India. She has 7 years of Industry experience and 12 years of academic experience. Her area of interest is in data mining and text mining and has published articles in various International Journals

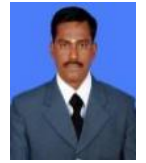

Dr. K. KARTHEEBAN working as Associate professor, Department of computer Science and Engineering, Kalasalingam Academy of Research and Education, Krishnankoil, Tamilnadu, India. Also he was worked as a Deputy Director Academic. He received his M.E degree in computer science and Engineering from College of Engineering, Anna University, and Chennai in 2007 and Completed $\mathrm{PhD}$ in the title of "Development of Efficient Algorithms for Secure Communication in Distributed Computing Environment" in the Department of Computer Science and Engineering at Kalasalingam Academy of Research and Education, Anandnagar, and TAMILNADU, INDIA in 2014. He worked as a faculty from Adhiyamaan College of Engineering, Hosur, India between 1996-1998. Currently 1 scholar completed his $\mathrm{PhD}$ and 6 students are doing their $\mathrm{PhD}$ under him with the topics such as Internet of Things, Medical Image Processing, Video Analytics, Cyber Forensics, Sentimental Analysis and Scheduling in Cloud computing. He has published many papers in SCI journals and Scopus indexed conferences. Also he has submitted proposals to DeIT, SERB and DRDO. His areas of interests include IoT, Medical image processing, cryptography and bioinformatics and grid and cloud computing. 